\title{
Biodegradability assessments of organic substances and polymers
}

\author{
Gérald Thouand
}

Received: 18 January 2014 / Accepted: 14 February 2014 / Published online: 17 May 2014

(C) Springer-Verlag Berlin Heidelberg 2014

The biodegradation of organic substances is an undoubtedly natural process; it is so common that we usually forget how hard it is to assess. Official references define the biodegradation process as 'the biologically mediated degradation or transformation of chemicals usually carried out by microorganisms' (ECHA, 2012). The International Union of Pure and Applied Chemistry (IUPAC) widens the definition to include the breakdown of a substance in vitro or in vivo as catalysed by enzymes (IUPAC, 1993).

The assessment of biodegradation (i.e. the biodegradation test or assay) can be conducted in many ways depending on the objective of the study. Biodegradability assays are designed to batch test a chemical substance (or polymer) as the sole carbon source in a mixed inoculum from different origins (river water, seawater, activated sludge and soil). Monitoring the biodegradation process remains a difficult task, ranging from the use of very basic sensors (oxygen or carbon dioxide) to very sophisticated analysis (Raman spectroscopy, nuclear magnetic resonance, etc.). At this stage of the assessment process, the data collected after considering the biodegradation kinetics are classified in two ways. The first is to claim that the substance is degraded by a biological process (biodegradable), and the second is to predict the persistence of the substance in the environment using the collected data. This classification assumes that the test would mimic one part of a complex environmental process. The European Registration, Evaluation, Authorisation and Restriction of Chemicals (REACH) regulation envisions a tiered approach to evaluating persistence. The first tier (or screening level) includes the use

Responsible editor: Philippe Garrigues

G. Thouand $(\bowtie)$

University of Nantes, UMR CNRS 6144 GEPEA, Institute for

Technology, La Roche sur Yon, France

e-mail: gerald.thouand@univ-nantes.fr of a cheap and simple test of ready biodegradability corresponding to Organisation for Economic Co-operation and Development (OECD) guidelines (no. $301 \mathrm{~A}$ to $\mathrm{F}$ ). This test consists of a screen for chemicals that are of no great concern in terms of environmental persistence. A non-readily-biodegradable substance is considered persistent unless its environmental degradability is supported by more expensive and complex simulation tests, as described in OECD guidelines (nos. 303, 307, 308 and 309). The type of simulation test(s) to be performed depends on the potential receptor environments of concern (wastewater treatment plants, surface water, sediment or soil).

With approximately 19,710 articles (or 20,928 including 2013), there has been a respectable quantity of scientific production. The first study was registered in 1960 (Kagawa et al. 1960), but biodegradation studies began more than a century ago (Fig. 1). The subject of biodegradation assessment was first addressed by Borstlap and Kooijman (1963). Since then, no less than 7,442 articles have addressed biodegradation testing (which represent $35 \%$ of all the articles published on biodegradation), and the REACH regulation process that started in 2001 has played a major role in the exponential production of articles displayed in Fig. 2.

This group of publications primarily addressed the inocula used in the tests as well as the design of new devices and the interpretation of biodegradation kinetics. These contributions provided many answers, but some questions remain open and the inocula, the sizes of the tests and the sensor devices are still motivating issues for many research teams. However, the challenges posed by mixtures of organic pollutants and organic-inorganic compounds will certainly supply many future scientific contributions because the subject, which exists at the interface of several disciplines (microbiology, material science engineering, chemistry and statistics), is quite complex. 
Fig. 1 Publication production from 1960 to 2012 (ISI Web of Science, key words: BIODEGRADA* and key words: BIODEGRADA* AND ASSAYS* OR TEST* OR METHOD* OR EVALUATION*)
Fig. 2 Distribution of the 7,442 publications produced in relation to biodegradation assessment, with a sharp increase indicated from 2004 to 2013

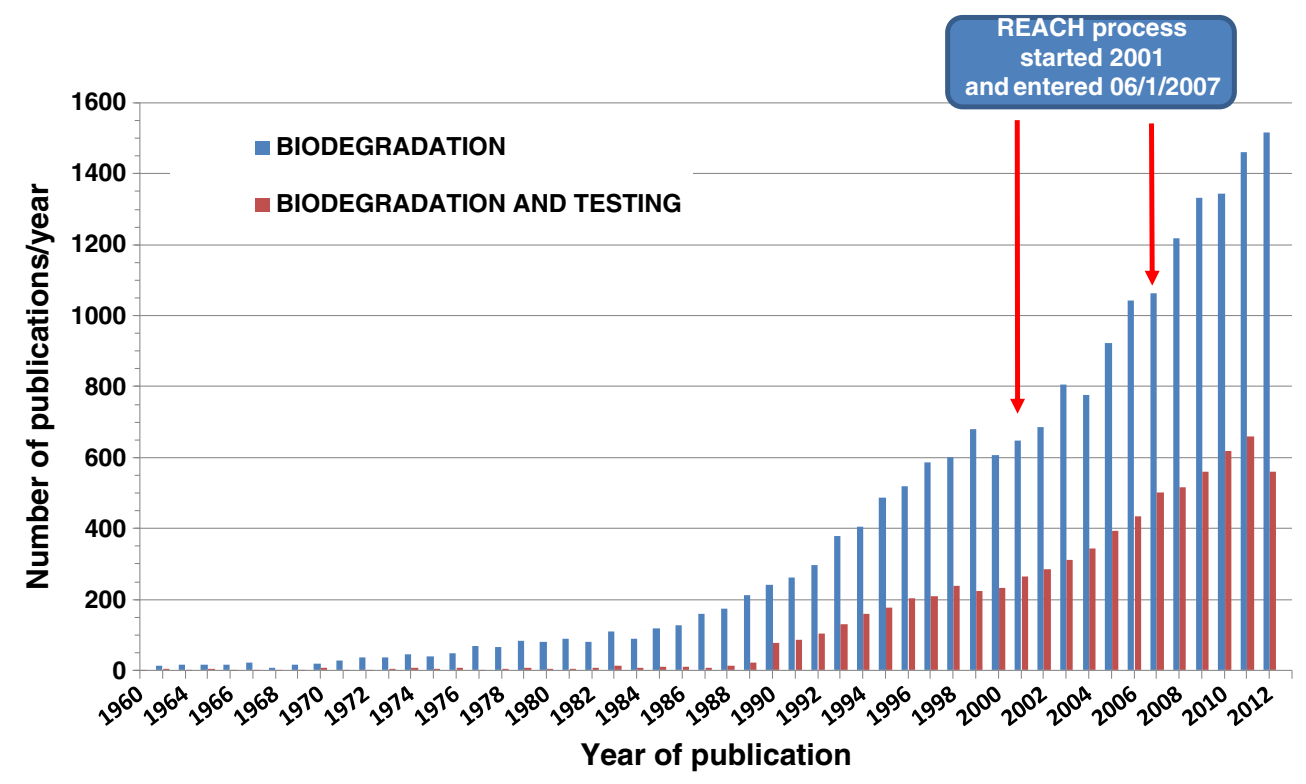

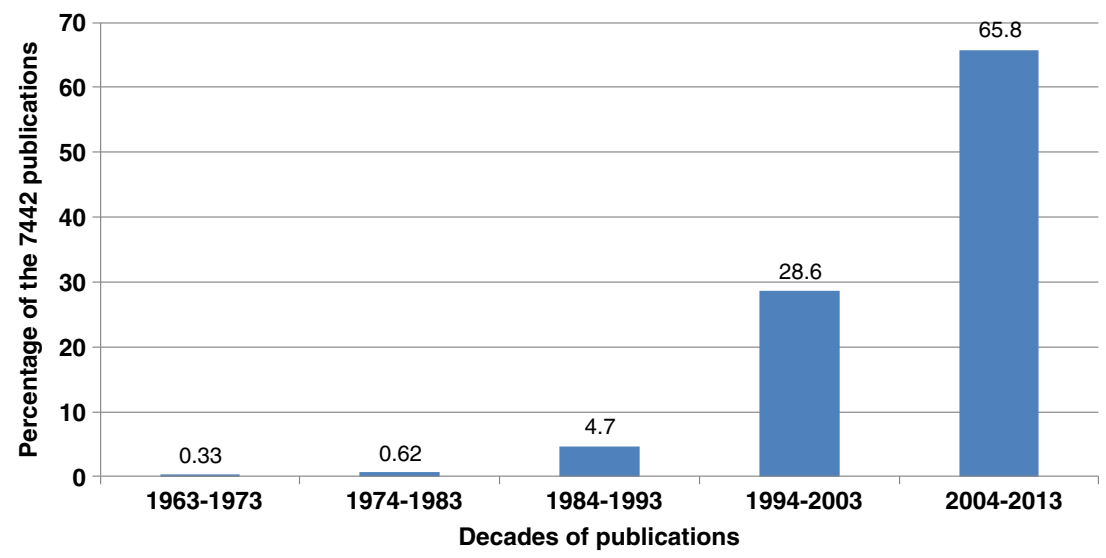

I would like to thank all the authors for their excellent contributions, and I would also like to thank the ESPR editing team for their valuable support throughout the preparation of this special issue.

\section{References}

Borstlap C, Kooijman PL (1963) A study of biodegradation of anionic synthetic detergents a new laboratory test. J Am Oil Chem Soc 40: 78-80.

European Chemicals Agency (2012) Guidance on information requirements and chemical safety assessment. Chapter R.7b: Endpoint specific guidance November 2012:159.

IUPAC (1993) Glossary for chemists of terms used in toxicology (IUPAC Recommendations 1993): 2020.

Kagawa Y, Mano Y, Shimazono N (1960) Biodegradation of dehydro-Lascorbic acid-2,3-diketo-aldonic acid decarboxylase from rat liver. Biochem Biophys Acta 43:348-349.

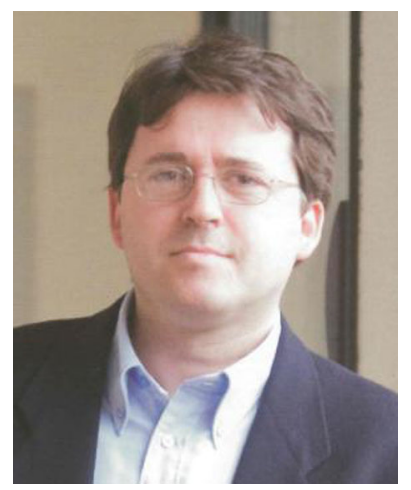

Gérald Thouand received his $\mathrm{PhD}$ degree in Microbiology from the University of Nancy, France, in 1993. He is full professor in microbiology at the University of Nantes, deputy for research innovation at the French Ministry of Research (DRRT Pays de La Loire) and auditor at the Institute of Higher Studies for Science and Technology (IHEST, Paris).

His research interests include environmental monitoring of biodegradation and biotechnology using microbial biosystems. He is mainly involved in the development of biosensors for chemical pollutant detection and pathogenic bacteria. He is a member of the French Society of Microbiology (SFM, France) and the Society for Applied Microbiology (SFAM, UK). He is associate editor at Frontiers in Microbiology (Frontiers in Microbial Ecotoxicology and Bioremediation), editor for Environmental Science and Pollution Research, Springer Verlag_-guest editor of Analytical Bioanalytical Chemistry (special issue 'Microorganisms for analysis') and member of the scientific committee of the International Society of Bioluminescence and Chemiluminescence. 